\title{
Stereotactic management of arrhythmia - radiosurgery in treatment of ventricular tachycardia (SMART-VT) - clinical trial protocol and study rationale
}

\author{
Marcin Miszczyk ${ }^{1}$, Tomasz Jadczyk ${ }^{2,3}$, Bartłomiej Tomasik, , Tomasz Latusek ${ }^{5}$,

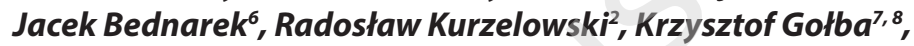 \\ Wojciech Wojakowski ${ }^{2}$, Krystian Wita ${ }^{9}$, Łukasz Dolla $^{10}$, Aleksandra Grzqdziel ${ }^{10}$, \\ Sławomir Blamek \\ 'IIIDepartment of Radiotherapy and Chemotherapy, Maria Sklodowska-Curie National Research Institute \\ of Oncology, Gliwice, Poland \\ ${ }^{2}$ Department of Cardiology and Structural Heart Diseases, Medical University of Silesia, Katowice, Poland \\ ${ }^{3}$ Interventional Cardiac Electrophysiology Group, International Clinical Research Center, St. Anne's \\ University Hospital Brno, Brno, Czech Republic \\ ${ }^{4}$ Department of Biostatistics and Translational Medicine, Medical University of Lodz, Lodz, Poland \\ ${ }^{5}$ Department of Radiotherapy, Maria Sklodowska-Curie National Research Institute of Oncology, Gliwice, \\ Poland \\ ${ }^{6}$ Department of Electrocardiology, John Paul II Hospital, Cracow, Poland \\ ${ }^{7}$ Department of Electrocardiology, Upper-Silesian Heart Center, Katowice, Poland \\ ${ }^{8}$ Department of Electrocardiology and Heart Failure, Medical University of Silesia, Katowice, Poland \\ ${ }^{9}$ First Department of Cardiology, Medical University of Silesia, Katowice, Poland \\ ${ }^{10}$ Department of Radiotherapy Planning, Maria Sklodowska-Curie National Research Institute of Oncology, \\ Gliwice, Poland
}

Correspondence:

Stawomir Blamek

Maria Sklodowska-Curie National

Research Institute of Oncology 44-102 Gliwice, ul. Wybrzeże Armii Krajowej 15 phone: +48322 788052 e-mail: slawomir.blamek@io.gliwice.pl

Received: 29.12.2020 Accepted:

1.02.2021

DOI: 10.24292/01.OR.420010221 Copyright $\odot$ Medical Education. All rights reserved.

\section{ABSTRACT}

Patients with ventricular tachycardia are usually treated with antiarrhythmic drugs and ablation if the arrhythmia substrate is available for invasive treatment. Despite high efficiency of this treatment there is a significant group of patients who do not benefit from available treatment methods, either because they cannot be applied or do not allow for durable control of the disease. For that reason a novel treatment method, STereotactic Arrhythmia Radioablation (STAR) has been proposed and its safety and efficiency is extensively studied throughout the world. The method is based on irradiation of the arrhythmia substrate identified with electrophysiological examination with high-precision image-guided radiosurgical methods usually used for ablation of malignant tumors. Here we present the protocol of the first Polish study on STAR in patients with intractable ventricular tachycardia (STARVT, NCT04642963), designed to test the safety of the method. Secondary endpoints include measures of the treatment efficiency.

Key words: ventricular tachycardia, ablation, radiosurgery, STAR, arrhythmia 


\section{INTRODUCTION}

Since its first application in October 2012, radiotherapy in the treatment of ventricular tachycardia (VT) has proven its effectiveness through case reports, clinical series and prospective trials [1]. The rapid development of STereotactic Arrhythmia Radioablation (STAR) was driven by a relatively large subset of patients with unmet medical needs at that time. Radiofrequency catheter ablation is a standard treatment strategy for VT being associated with up to $50 \%$ recurrence rate at 6 months and decreasing efficacy with every subsequent ablation [2]. In such patients, STAR can be effective despite prior treatment failures, providing alternative clinical solution. The first Polish prospective clinical trial presented in this article aims to confirm the safety of STAR, establish cooperation between radiation oncologists, cardiologists and electrophysiologists, and provide framework for future studies.

\section{TRIAL METHODOLOGY}

This is a prospective, two-center, single-arm study. Patients with a medical history of sustained or recurrent VT despite previous catheter ablation procedures, or presenting with contraindications to catheter ablation, will be treated with single-fraction radiosurgery of $25 \mathrm{~Gy}$ to the arrhythmia substrate located with electrophysiological mapping. The trial aims to demonstrate the safety of the treatment method defined as 3-month observation without grade 3 or higher adverse events (CTCAE v5.0) in at least 6 out of 7 patients in the $1^{\text {st }}$ stage of the study, and in total in at least 9 out of 11 patients ( $2^{\text {nd }}$ stage) with an interim safety analysis of primary outcome data in 7 consecutively enrolled patients. After the initial period, the patients will be monitored every 3 months until $12^{\text {th }}$ month, and every 6 months thereafter. Secondary aims include assessment of clinical efficacy (reduction of VT burden and improvement in patient reported outcomes), changes in the uptake of antiarrhythmic medications, dynamics of myocardial injury biomarkers, and overall survival and cause-specific survival.

\section{TRIAL REGISTRATION}

The study is registered in the ClinicalTrials.gov database of the National Institute of Health - U.S. National Library of Medicine, under the name Stereotactic Management of Arrhythmia - Radiosurgery in Treatment of Ventricular Tachycardia (SMART-VT) and received clinical trial identifier NCT04642963.

\section{TRIAL FUNDING}

This is a non-commercial clinical trial supported by the courtesy of Maria Sklodowska-Curie National Research Institute of On- cology, Gliwice Branch, Poland and Professor Leszek Giec Upper Silesian Medical Center of the Medical University of Silesia in Katowice, Poland. The authors and investigators receive no remuneration for the participation in this trial.

\section{BRIEF HISTORY OF STAR}

The cardiac ablation radiosurgery was set in motion a decade ago by pre-clinical studies conducted within the CyberHeart ${ }^{\mathrm{TM}}$ project which determined that a dose of 25-35 Gy is capable of producing fibrotic lesions, similar to those induced by catheter ablation $[3,4]$. Despite an ongoing dispute regarding the actual mechanism of action of ionizing radiation on the ventricular myocardium, the findings were soon translated into clinic through the first-in-human applications in 2012 in the US by Loo et al. [5], followed shortly by Cvek et al. [6] in Europe. Up to date, a number of clinical applications have been described in the literature [5-21], including results of two prospective clinical trials. The first study, published by Robinson et al. [12], demonstrated both safety and effectiveness of this method. The authors reported no serious acute adverse effects, and treatment related grade $\geq 3$ toxicity developed in only 2 out of 19 patients. The $50 \%$ and $95 \%$ reduction in VT episodes were achieved in $94 \%$ and $61 \%$ of the patients, respectively. On the other hand, in the study by Gianni et al. [13], despite the favourable safety profile, the efficacy was suboptimal and longterm arrhythmia control was not achieved, similarly to the recently published retrospective case series by Chin et al. [11].

\section{STUDY RATIONALE}

Although majority of literature data is in favour of STAR, the inconsistent results of the recent clinical trial [13] and retrospective report [11] warrant further investigation. Considering STAR has never yet been performed in Poland and treatment techniques are significantly different between institutions and authors, a pilot trial focused on treatment safety was chosen to be the most appropriate.

\section{STATISTICAL ASSUMPTIONS}

The primary endpoint is to assess the post-intervention safety defined as no treatment-related serious adverse events (grade $\geq 3$ according to (TCAE v5.0) in the first 90 days after radiotherapy. The sample size planning is based on the assumption that observed safety of $<50 \%$ (null hypothesis) will lead to the rejection of the alternative hypothesis of a safety of $>90 \%\left(\mathrm{H}_{1}\right)$. If the safety is in between, the statement will be confined to the confidence interval. We assumed a level of $2.5 \%$ (one-sided) and a power of $80 \%(\beta=0.2)$. 
Based on Simon and Fleming's two-stage designs [22, 23], an interim analysis is performed after the first seven included patients have been assessed for the primary endpoint (fig. 1). The inclusion of additional patients cannot be carried out until at least 90 days after the completion of treatment of the seventh patient. The study will be terminated early if an endpoint occurs in more than one patient in this group. Otherwise, an additional four patients will be enrolled and the total number of patients will be eleven. To reject the nul hypothesis, no more than two grade $\geq 3$ events can occur.
- prior radiotherapy to the thoracic region (relative contraindication)

- failure to induce VT during electrophysiological study.

\section{WORK-UP AND TREATMENT DELIVERY}

The procedural workflow is briefly presented on figure 2 .

FIGURE 1. Study design.

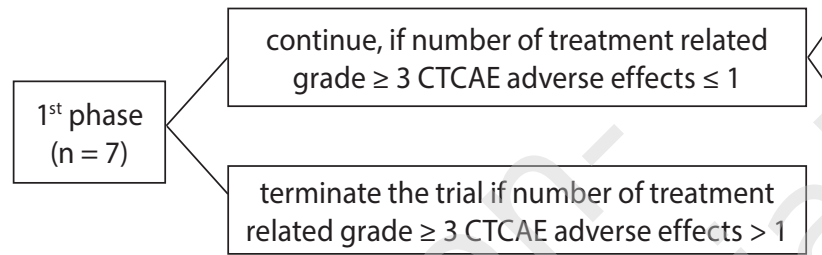

\section{INCLUSION AND EXCLUSION CRITERIA}

The study includes patients of 18 years or older, which meet the following criteria:

- Patients with structural heart disease and implantable cardioverter defibrillator (ICD).

- Clinically significant arrhythmia with at least 3 VT episodes per month despite adequate pharmacological treatment.

- At least one episode of monomorphic VT registered during electrophysiological study.

- Recurrent VT despite at least one prior catheter ablation and adequate pharmacotherapy OR contraindications to catheter ablation and/or pharmacotherapy (i.e., patient with medically contraindicated catheter ablation is obliged to undergo only pharmacotherapy prior to study enrolment).

- Patient must be able to understand and be willing to sign a written informed consent document.

The patient must not meet the following exclusion criteria:

- heart failure requiring inotropic treatment or mechanical assistance

- arrhythmia due to cardiac channelopathy

- reversible source of arrhythmia

- NYHA (New York Heart Association) stage IV heart failure

- myocardial infarction or cardiac surgery within last 3 months

- life expectancy $<6$ months

- polymorphic VT

- pregnancy the safety is confirmed if number of treatment related grade $\geq 3$ CTCAE adverse effects is $\leq 2$ in a total of 11 patients treated

safety insucfficient if $>2$ treatment related grade $\geq 3$ CTCAE adverse effects

FIGURE 2.

Procedural workflow.

\section{StereoTactic Arrhythmia Radioablation (STAR)}

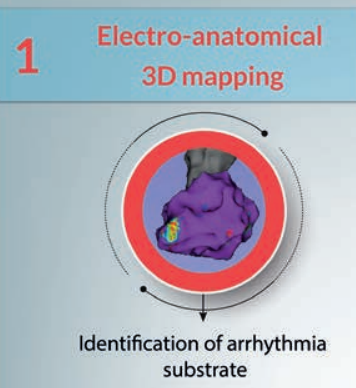

3 Target delineation

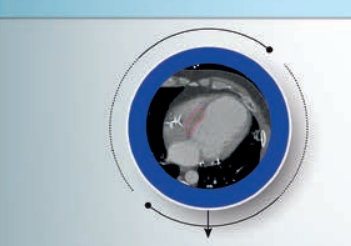

Contouring of the $\mathrm{CT}$ structure corresponding to the arrhythmia substrate CTintegration $\quad 2$

5

Stereotactic radioablation treatment

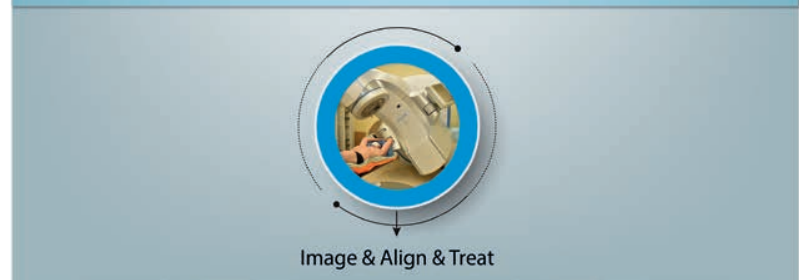


The radiotherapy planning procedures start with preparation of individual immobilization device (vacuum bag). Then, deep inspiration breath hold (DIBH) or respiratory-gated non-contrast enhanced treatment planning computer tomography (CT) is performed, followed by contrast-enhanced diagnostic thoracic CT.

Next, the patient is admitted to the cardiology ward for catheter-based electro-anatomical study of the left ventricle (LV) and/or right ventricle (RV) using EnSite Precision intracardiac system. Three-dimensional (3D) reconstruction of LV/RV combined with high-density endocardial map allows for precise identification of the healthy and diseased myocardium as well as fibrotic tissue characterized by bipolar voltage of $>1.5 \mathrm{mV}$, $1.5-0.5 \mathrm{mV}$ and $<0.5 \mathrm{mV}$, respectively. Selection of arrhythmogenic areas is based on 3D color-coded voltage map delineating diseased/scar tissue border. Additionally, programmed ventricular stimulation is used to confirm inducibility of sustained monomorphic VT.

The location of the arrhythmia substrate is transferred to the contrast-enhanced CT through the mutual effort of electrophysiologist, cardiologists and radiation oncologist involved in the treatment. The precise delineation of the treatment volume is crucial. The process is aided by defining the involved heart segment as described previously [24]. The location of the target volume for radiotherapy can be additionally compared to the results of fusion of the EP data and CT images performed with Slicer 3D software run with an extension developed by Hohmann et al. as a double-check procedure for target delineation [25].

The radiotherapy planning is carried out using Varian ECLIPSE ${ }^{\text {TM }}$ treatment planning system (TPS) and VMAT (volumetric modulated arc therapy) technology with either DIBH or respiratory gating, using dose constraints presented in table 1. Most of these values are adopted from thoracic stereotactic radiotherapy (i.e. targeted at primary and metastatic lesions in lungs or skeleton), with the exception of coronary arteries. The dose constraint for coronary arteries was chosen through extrapolation of available data using the principle of maximum safety, and is prone to change when additional data on safety is available. The choice of the treatment technique depends on the patient's ability to hold breath for time required to deliver the dose. If breath-hold technique cannot be applied, the dose is delivered during free breathing and the operation of the linear accelerator is gated with patient's breath. The treatment can be performed either with Varian $\mathrm{EDGE}^{\mathrm{TM}}$ machine allowing for short treatment time and offering gated $C B C T$ image verification prior to treatment, or with the Accuray CyberKnife ${ }^{\mathrm{TM}}$ platform. The latter can also produce excellent STAR radiotherapy plans [26] and, although is not capable of CBCT imaging, it can track the position of the ICD lead during irradiation which in fact becomes a fiducial marker for radiotherapy. Along with the Synchrony ${ }^{\circledR}$ automatic real-time motion synchronization and tracking system it allows for effective respiratory motion management without the need of gated radiation delivery.

TABLE 1.

Dose constraints.

\begin{tabular}{|c|c|c|c|}
\hline OAR & Volume & Volume dose & Point dose* \\
\hline PTV minus CTV & - & - & $31.25 \mathrm{~Gy}$ \\
\hline CTV & $<1 \mathrm{~cm}^{3}$ & $32.5 \mathrm{~Gy}$ & $14 \mathrm{~Gy}$ \\
\hline \multirow{2}{*}{ Spinal cord } & $<0.35 \mathrm{~cm}^{3}$ & $10 \mathrm{~Gy}$ & $15.4 \mathrm{~Gy}$ \\
\cline { 2 - 4 } & $<1.2 \mathrm{~cm}^{3}$ & $8 \mathrm{~Gy}$ & $22 \mathrm{~Gy}$ \\
\hline Esophagus & $<5 \mathrm{~cm}^{3}$ & $11.9 \mathrm{~Gy}$ & $17 \mathrm{~Gy}$ \\
\hline Stomach & $<5 \mathrm{~cm}^{3}$ & $17.4 \mathrm{~Gy}$ & $20.2 \mathrm{~Gy}$ \\
\hline Duodenum & $<5 \mathrm{~cm}^{3}$ & $11.2 \mathrm{~Gy}$ & \\
\hline Trachea and main bronchi & $<10 \mathrm{~cm}^{3}$ & $9 \mathrm{~Gy}$ & \\
\hline Lungs (together) & $<4 \mathrm{~cm}^{3}$ & $17.4 \mathrm{~Gy}$ & \\
& & & $7 \mathrm{~Gy}$ \\
\hline Liver & $<1500 \mathrm{~cm}^{3}$ & $7.6 \mathrm{~Gy}$ & \\
\hline Kidneys (together) & $<1000 \mathrm{~cm}^{3}$ & $8 \mathrm{~Gy}$ & $11 \mathrm{~Gy}$ \\
\hline Coronary arteries & $<37 \%$ & $9.5 \mathrm{~Gy}$ & $33 \mathrm{~Gy}$ \\
\hline Ribs & $<700 \mathrm{~cm}^{3}$ & - & $27.5 \mathrm{~Gy}$ \\
\hline Skin & $<200 \mathrm{~cm}^{3}$ & $25.5 \mathrm{~Gy}$ & \\
\hline
\end{tabular}

* Defined as dose in $<0.035 \mathrm{cc}$. ^ Left coronary artery including anterior intraventricular and circumflex, and right coronary artery including posterior descending artery. 
The treatment is performed in the assistance of the responsible cardiologist, with appropriate ICD management and continuous cardiac monitoring during radiotherapy delivery [27]. The patient is admitted to the cardiology ward a day prior, and transported back to the hospital after the procedure.

\section{FOLLOW-UP}

The follow-up schedule is presented in table 2. The ICD readouts serve as a measure to assess the VT burden along with the electrocardiography examination (ECG). Holter ECG monitoring performed after RT provides additional information regarding immediate anti-arrhythmic effect of the treatment. As the VT burden decreases, the attending physician is encouraged to decrease the anti-arrhythmic drugs therapy, and the current dose is recorded at every visit.

Due to the fact that majority of the patients present with heart failure with reduced ejection fraction (HFrEF), often with left ventricle ejection fraction (LVEF) of 20-30\%, echocardiography is crucial for monitoring of the treatment toxicity along with the assessment of adverse effects using CTCAE v5.0 scale. Moreover, the heart failure severity is assessed with NYHA scale, and patient reported outcomes are measured through EuroQol EQ-5D ques- tionnaire. Laboratory tests - creatine kinase, cardiac $\mathrm{T}$ troponin, $\mathrm{N}$-terminal prohormone B-type natriuretic peptide (NT-proBNP) serve as an additional index of myocardial injury.

The optional examination includes follow-up CT and MRI. Generally, due to significant comorbidities presented by the patients, often including renal failure, we leave the choice to the attending physician discretion.

\section{OTHER CONSIDERATIONS}

The cooperation between radiotherapy and cardiology departments extending far beyond the usual safety or treatment side effects management issues brings a multitude of new challenges and difficulties. Despite proper theoretical work-up and practical training in centers experienced in cardiac radiosurgery, our team has found dozens of unexpected obstacles, and it took us many mock cases to overcome those. A number of issues starting from proper imaging, through integration of electrophysiological data, appropriate patient setup and target tracking during treatment had to be worked out and solved. We encourage physicians who consider using STAR to contact the corresponding author for further information. We have started enrolment and the first patient was treated in December 2020.

TABLE 2.

Follow-up schedule.

\begin{tabular}{|c|c|c|c|c|c|c|c|c|c|c|}
\hline & pre-RT & post-RT & $\mathbf{1} \mathbf{w k}$ & $\mathbf{6} \mathbf{w k}$ & $\mathbf{3} \mathbf{m}$ & $\mathbf{6} \mathbf{m}$ & $\mathbf{9} \mathbf{m}$ & $\mathbf{1 2} \mathbf{m}$ & $\mathbf{1 8} \mathbf{m}$ & $\mathbf{2 4} \mathbf{m}$ \\
\hline Clinical examination & $\mathrm{X}$ & $\mathrm{X}$ & $\mathrm{X}$ & $\mathrm{X}$ & $\mathrm{X}$ & $\mathrm{X}$ & $\mathrm{X}$ & $\mathrm{X}$ & $\mathrm{X}$ & $\mathrm{X}$ \\
\hline ECG & $\mathrm{X}$ & & & $\mathrm{X}$ & $\mathrm{X}$ & $\mathrm{X}$ & $\mathrm{X}$ & $\mathrm{X}$ & $\mathrm{X}$ & $\mathrm{X}$ \\
\hline Holter ECG & & $\mathrm{X}$ & & & & & & & & \\
\hline Echocardiography & $\mathrm{X}$ & & & & $\mathrm{X}$ & & & $\mathrm{X}$ & & $\mathrm{X}$ \\
\hline ICD readout & $\mathrm{X}$ & & & $\mathrm{X}$ & $\mathrm{X}$ & $\mathrm{X}$ & $\mathrm{X}$ & $\mathrm{X}$ & $\mathrm{X}$ & $\mathrm{X}$ \\
\hline AE- CTCAE v5.0 & $\mathrm{X}$ & $\mathrm{X}$ & $\mathrm{X}$ & $\mathrm{X}$ & $\mathrm{X}$ & $\mathrm{X}$ & & & & $\mathrm{X}$ \\
\hline Drug uptake assessment & $\mathrm{X}$ & $\mathrm{X}$ & $\mathrm{X}$ & $\mathrm{X}$ & $\mathrm{X}$ & $\mathrm{X}$ & $\mathrm{X}$ & $\mathrm{X}$ & $\mathrm{X}$ & $\mathrm{X}$ \\
\hline NYHA, EuroQol EQ-5D & $\mathrm{X}$ & $\mathrm{X}$ & $\mathrm{X}$ & $\mathrm{X}$ & $\mathrm{X}$ & $\mathrm{X}$ & $\mathrm{X}$ & $\mathrm{X}$ & $\mathrm{X}$ & $\mathrm{X}$ \\
\hline Laboratory tests & $\mathrm{X}$ & & $\mathrm{X}$ & $\mathrm{X}$ & $\mathrm{X}$ & & & $\mathrm{X}$ & & $\mathrm{X}$ \\
\hline $\mathrm{CT}$ & $\mathrm{X}$ & & & & $\mathrm{O}$ & & & $\mathrm{O}$ & & $\mathrm{O}$ \\
\hline MRI & $\mathrm{O}$ & & & & $\mathrm{O}$ & & & $\mathrm{O}$ & & $\mathrm{O}$ \\
\hline
\end{tabular}

O- optional. 


\section{References}

1. Lydiard S, Hugo G, O'Brien R et al. A review of cardiac radioablation (CR) for arrhythmias: procedures, technology and future opportunities. Int J Radiat Oncol.; 0. Epub ahead of print November 2020. http://doi.org/10.1016/j.jirobp.2020.10.036.

2. Tokuda M, Kojodjojo P, Tung S et al. Acute failure of catheter ablation for ventricular tachycardia due to structural heart disease: Causes and significance. J Am Heart Assoc. Epub ahead of print 2013. http://doi.org/10.1161/JAHA.113.000072.

3. Sharma A, Wong D, Weidlich $\mathrm{G}$ et al. Noninvasive stereotactic radiosurgery (CyberHeart) for creation of ablation lesions in the atrium. Hear Rhythm. 2010; 7: 802-10. https://doi.org/10.1016/j.hrthm.2010.02.010.

4. Maguire PJ, Gardner E, Jack AB et al. Cardiac Radiosurgery (CyberHeart ${ }^{\mathrm{TM}}$ ) for Treatment of Arrhythmia: Physiologic and Histopathologic Correlation in the Porcine Model. Cureus. Epub ahead of print 24 July 2012. http://doi.org/10.7759/cureus.32.

5. Loo BW, Soltys SG, Wang L et al. Stereotactic Ablative Radiotherapy for the Treatment of Refractory Cardiac Ventricular Arrhythmia. Circ Arrhythmia Electrophysiol. 2015; 8: 748-50. https://doi.org/10.1161/CIRCEP.115.002765.

6. Cvek J, Neuwirth R, Knybel L et al. Cardiac Radiosurgery for Malignant Ventricular Tachycardia. Cureus. Epub ahead of print 22 July 2014 . http://doi. org/10.7759/cureus.190.

7. Jumeau R, Ozsahin M, Schwitter J et al. Rescue procedure for an electrical storm using robotic non-invasive cardiac radio-ablation. Radiother Oncol 2018; 128: 189-91. https://doi.org/10.1016/j.radonc.2018.04.025.

8. Cuculich PS, Schill MR, Kashani R et al. Noninvasive Cardiac Radiation for Ablation of Ventricular Tachycardia. N Engl J Med. Epub ahead of print 2017. http://doi.org/10.1056/NEJMoa1613773.

9. Neuwirth R, Cvek J, Knybel L et al. Stereotactic radiosurgery for ablation of ventricular tachycardia. EP Eur. 2019; 21: 1088-95. https://doi.org/10.1093/ europace/euz133.

10. Lloyd MS, Wight J, Schneider F et al. Clinical experience of stereotactic body radiation for refractory ventricular tachycardia in advanced heart failure patients. Hear Rhythm. Epub ahead of print 1 October 2019. http://doi.org/10.1016/j.hrthm.2019.09.028.

11. Chin R, Hayase J, Hu P et al. Non-invasive stereotactic body radiation therapy for refractory ventricular arrhythmias: an institutional experience. J Interv Card Electrophysiol. Epub ahead of print 2020. http://doi.org/10.1007/s10840-020-00849-0.

12. Robinson CG, Samson PP, Moore KMS et al. Phase I/II Trial of Electrophysiology-Guided Noninvasive Cardiac Radioablation for Ventricular Tachycardia. Circulation. 2019; 139: 313-21. https://doi.org/10.1161/CIRCULATIONAHA.118.038261.

13. Gianni C, Rivera D, Burkhardt JD et al. Stereotactic arrhythmia radioablation for refractory scar-related ventricular tachycardia. Hear Rhythm. Epub ahead of print 6 March 2020. http://doi.org/10.1016/j.hrthm.2020.02.036.

14. Haskova J, Peichl P, Pirk J et al. Stereotactic radiosurgery as a treatment for recurrent ventricular tachycardia associated with cardiac fibroma. Hear Case Reports. 2019; 5: 44-7. https://doi.org/10.1016/j.hrcr.2018.10.007.

15. Scholz EP, Seidensaal K, Naumann P et al. Risen from the dead: Cardiac stereotactic ablative radiotherapy as last rescue in a patient with refractory ventricular fibrillation storm. Hear Case Reports. 2019; 5: 329-32. https://doi.org/10.1016/j.hrcr.2019.03.004.

16. Zeng L, Huang L, Tan $\mathrm{H}$ et al. Stereotactic body radiation therapy for refractory ventricular tachycardia secondary to cardiac lipoma: A case report Pacing Clin Electrophysiol. 2019; 42: 1276-9. https://doi.org/10.1111/pace.13731.

17. Martí-Almor J, Jiménez-López J, Rodríguez de Dios $\mathrm{N}$ et al. Noninvasive ablation of ventricular tachycardia with stereotactic radiotherapy in a patient with arrhythmogenic right ventricular cardiomyopathy. Rev Esp Cardiol (Engl Ed). Epub ahead of print 30 July 2019. http://doi.org/10.1016/j. rec.2019.06.004.

18. Bhaskaran A, Downar E, Chauhan VS et al. Electroanatomical mapping-guided stereotactic radiotherapy for right ventricular tachycardia storm. Hear Case Reports 2019; 5: 590-2. https://doi.org/10.1016/j.hrcr.2019.09.007.

19. Krug D, Blanck O, Demming T et al. Stereotactic body radiotherapy for ventricular tachycardia (cardiac radiosurgery): First-in-patient treatment in Germany. Strahlentherapie und Onkol. 2020; 196: 23-30. https://doi.org/10.1007/s00066-019-01530-w.

20. Mayinger M, Kovacs B, Tanadini-Lang $S$ et al. First magnetic resonance imaging-guided cardiac radioablation of sustained ventricular tachycardia. Radiother Oncol. Epub ahead of print 2020. http://doi.org/10.1016/j.radonc.2020.01.008.

21. Park JS, Choi Y. Stereotactic cardiac radiation to control ventricular tachycardia and fibrillation storm in a patient with apical hypertrophic cardiomyopathy at burnout stage: Case report. J Korean Med Sci; 35. Epub ahead of print 13 July 2020. http://doi.org/10.3346/jkms.2020.35.e200.

22. Fleming TR. One-Sample Multiple Testing Procedure for Phase II Clinical Trials. Biometrics. Epub ahead of print 1982. http://doi.org/10.2307/2530297.

23. Simon R. Optimal two-stage designs for phase II clinical trials. Control Clin Trials. Epub ahead of print 1989. http://doi.org/10.1016/0197-2456(89)90015-9.

24. Cerqueira MD, Weissman NJ, Dilsizian V et al; American Heart Association Writing Group on Myocardial Segmentation and Registration for Cardiac Imaging. Standardized myocardial segmentation and nomenclature for tomographic imaging of the heart. A statement for healthcare professionals from the Cardiac Imaging Committee of the Council on Clinical Cardiology of the American Heart Association. Circulation. 2002; 105(4): 539-42. http://doi.org/10.1161/hc0402.102975.

25. Hohmann S, Henkenberens C, Zormpas C et al. A novel open-source software-based high-precision workflow for target definition in cardiac radioablation. J Cardiovasc Electrophysiol. Epub ahead of print 2020. http://doi.org/10.1111/jce.14660.

26. Wang L, Fahimian B, Soltys SG et al. Stereotactic Arrhythmia Radioablation (STAR) of Ventricular Tachycardia: A Treatment Planning Study. Cureus; 8. Epub ahead of print 15 July 2016. http://doi.org/10.7759/cureus.694.

27. Tajstra M, Blamek S, Niedziela JT et al. Patients with cardiac implantable electronic devices undergoing radiotherapy in Poland. Expert opinion of the Heart Rhythm Section of the Polish Cardiac Society and the Polish Society of Radiation Oncology. Kardiol Pol. 2019; 77(11): 1106-16. Epub 2019 Nov 19. http://doi.org/10.33963/KP.15063. 
Authors' contributions:

Marcin Miszczyk: study design, literature search, review and analysis, manuscript and figure preparation and review; Tomasz Jadczyk: study design, literature search, manuscript and figure preparation; Bartłomiej Tomasik: study design, development of study statistical rationale; Tomasz Latusek: study design, literature search, manuscript preparation; Jacek Bednarek: study design, manuscript preparation;

Radosław Kurzelowski: literature search and manuscript preparation; Krzysztof Gołba: manuscript review and supervision; Wojciech Wojakowski: study design, manuscript review and supervision; Krystian Wita: manuscript review and supervision; Łukasz Dolla: study design - irradiation technique and quality assurance, manuscript preparation; Aleksandra Grządziel: study design - irradiation technique and quality assurance, manuscript preparation; Sławomir Blamek: study design, manuscript preparation, review and supervision.

Conflict of interests:

Authors declare no conflict of interest.

Financial support: There was no financial support.

Ethics:

The authors had full access to the data and take full responsibility for its integrity. All authors have read and agreed with the content of the manuscript as written. The paper complies with the Helsinki Declaration, EU Directives and harmonized requirements for biomedical journals. 